Supporting Information for

\title{
Uniaxially Parallel Alignment of a Smectic A Liquid-Crystalline Rod-Coil Molecule and its Lithium Salt Complexes Using Rubbed Polyimides
}

Yosuke Iinuma, Kenji Kishimoto, Yoshimitsu Sagara, Masafumi Yoshio, Tomohiro Mukai, Ichiro Kobayashi, Hiroyuki Ohno, and Takashi Kato*

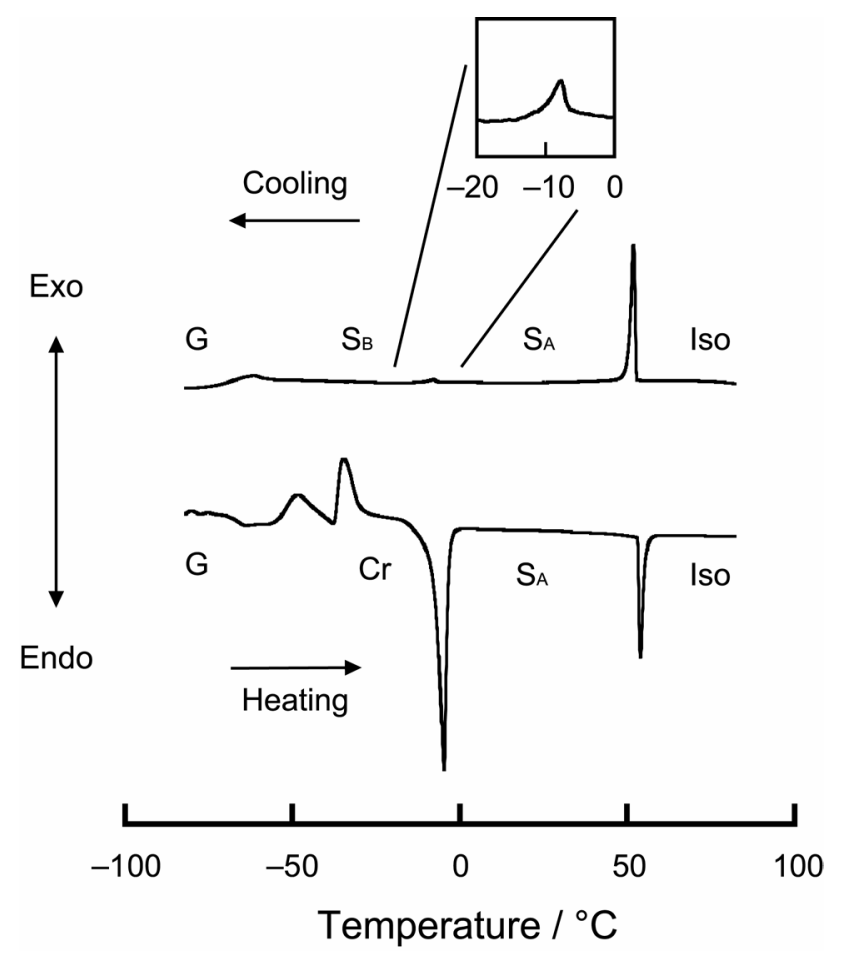

Figure S1. DSC thermograms of compound 1 at a scan rate of $10^{\circ} \mathrm{C} \mathrm{min}{ }^{-1}$. Iso: isotropic; $\mathrm{S}_{\mathrm{A}}$ : smectic A; $\mathrm{S}_{\mathrm{B}}$ : smectic B; G: glassy; Cr: crystalline. 
(a)

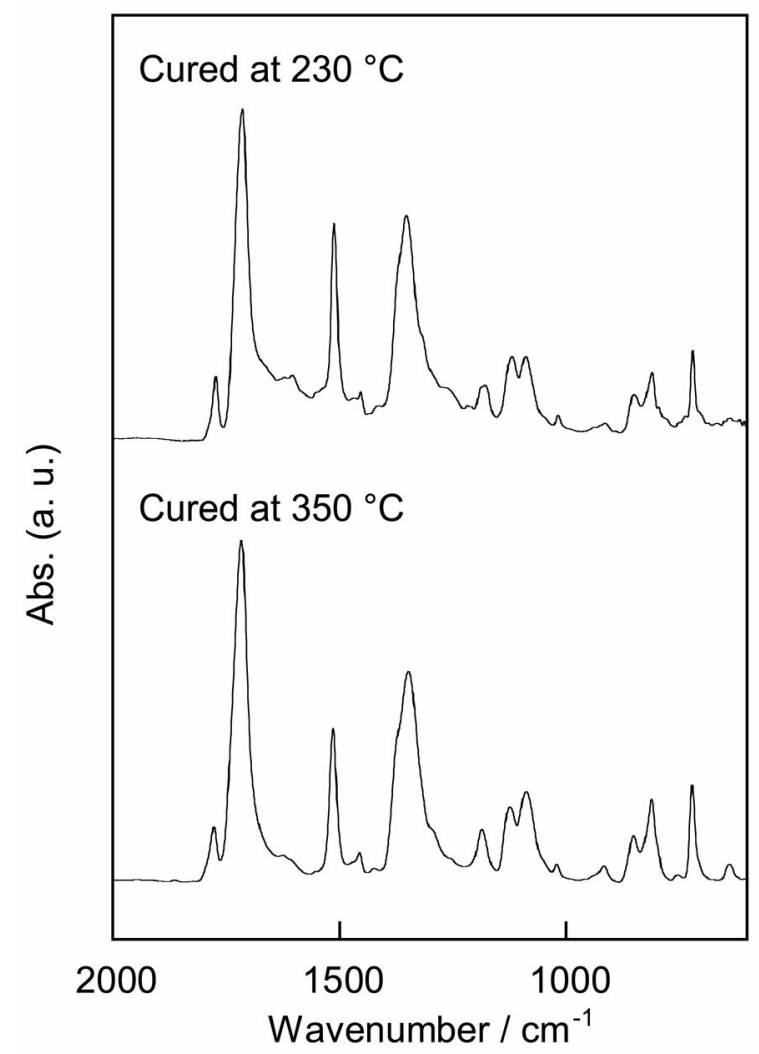

(b)

Cured at $230{ }^{\circ} \mathrm{C}$

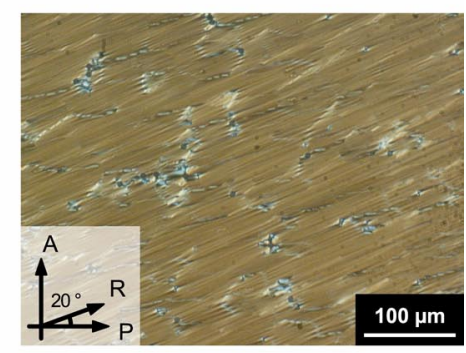

Cured at $350{ }^{\circ} \mathrm{C}$

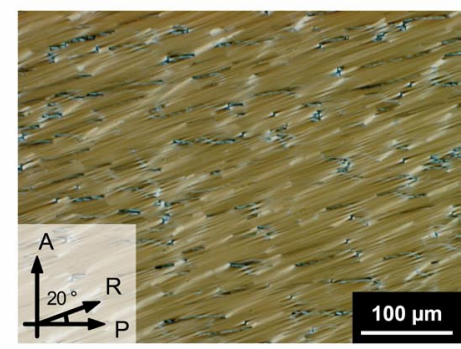

Figure S2. (a) IR spectra of polyimide 2 prepared by curing at 230 and $350{ }^{\circ} \mathrm{C}$ for 30 min and (b) polarizing optical microscopic images of $\mathbf{1}$ aligned parallel on these polyimide films of $\mathbf{2}$.

Polyimides 2-5 were obtained by curing at $230{ }^{\circ} \mathrm{C}$ for $\mathbf{2 - 4}$ and $200{ }^{\circ} \mathrm{C}$ for $\mathbf{5}$. The commercially available polyimides for the use as alignment layers for liquid crystal displays are normally cured around $200-250{ }^{\circ} \mathrm{C}$ in order to prevent the liquid-crystalline cells from damaging. However, the curing temperature may be low for the complete imidization. So, we prepared the polyimide films of $\mathbf{2}$ by curing at 230 and $350{ }^{\circ} \mathrm{C}$ and measured the infrared (IR) spectra to examine the influence of these polyimides on the alignment of the liquid crystals. As the results, no differences were seen in properties of alignment between polyimides obtained at the different temperatures. IR spectra of 2 obtained by curing at 230 and $350{ }^{\circ} \mathrm{C}$ are shown in Figure S2a. Absorption bands appeared at 1780, 1720, and 725 $\mathrm{cm}^{-1}$ are assigned to the $\mathrm{C}=\mathrm{O}$ stretching and bending in the imide group. The peak at $1380 \mathrm{~cm}^{-1}$ corresponds to the absorption of the $\mathrm{C}-\mathrm{N}$ stretching in the imide group. The degree of the imidization for the polymer obtained by curing at $230{ }^{\circ} \mathrm{C}$ was estimated to be $80 \%$ from the ratio of the imide band 
at $1380 \mathrm{~cm}^{-1}$ to the aromatic C-C stretching at $1500 \mathrm{~cm}^{-1}$ as a reference band if the imidization was completed for the polymer obtained at $350{ }^{\circ} \mathrm{C}$. ${ }^{1}$ Figure S2b shows the polarizing optical microscopic images of the liquid crystal aligned parallel on the surface of the rubbed polyimides prepared by curing 230 and $350{ }^{\circ} \mathrm{C}$. It was found that the use of polyimide films obtained by curing over $230{ }^{\circ} \mathrm{C}$ led to the uniaxially parallel alignment of liquid crystals and gave the same values of the contrast.

(a)

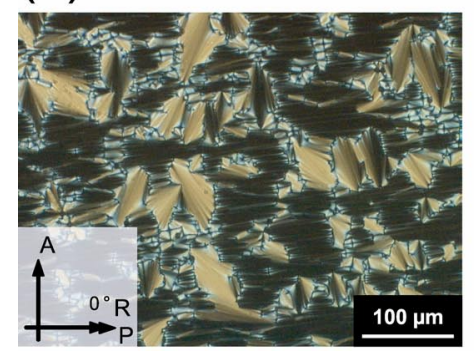

(b)

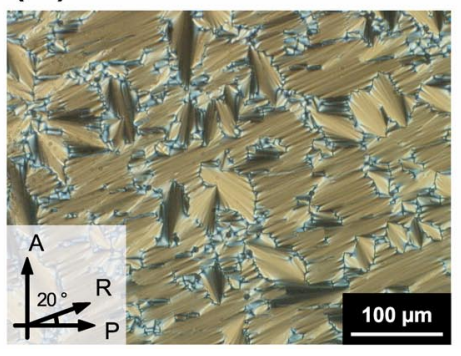

(c)

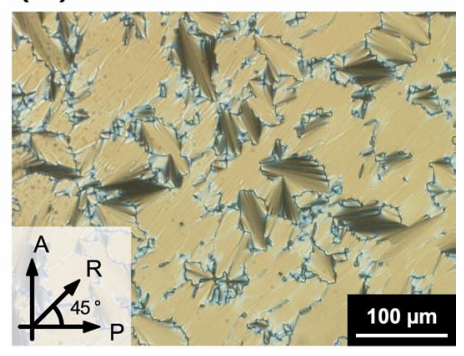

Figure S3. Polarizing optical microscopic images of the aligned LC compound $\mathbf{1}$ on polyimide $\mathbf{3}$. The rubbing direction is at the angle of (a) $0^{\circ}$ (b) $20^{\circ}$ (c) $45^{\circ}$ to polarizer or analyzer under crossed Nicols condition. Directions of A: analyzer; P: polarizer; R: rubbing.

(a)

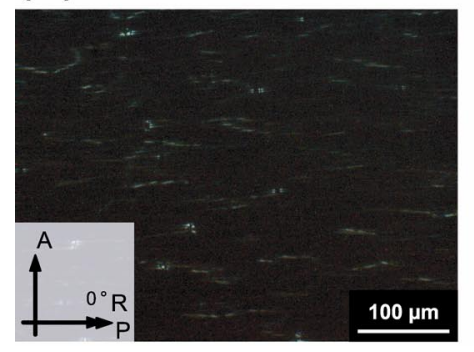

(b)

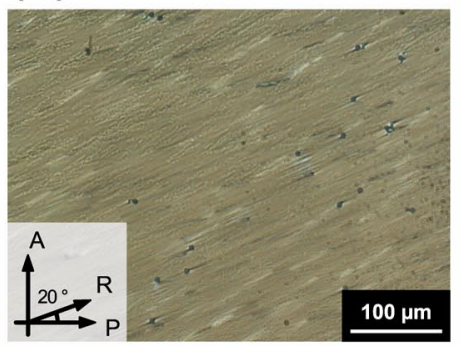

(c)

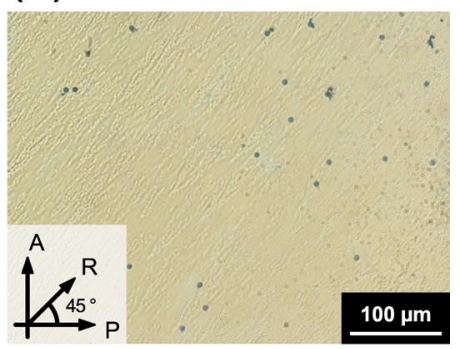

Figure S4. Polarizing optical microscopic images of the aligned complexes of 1 containing 0.05 mol of lithium triflate per ethylene oxide unit on polyimide 2 . The rubbing direction is at the angle of (a) $0{ }^{\circ}$ (b) $20^{\circ}$ (c) $45^{\circ}$ to polarizer or analyzer under crossed Nicols condition. Directions of A: analyzer; P: polarizer; R: rubbing. 
(a)

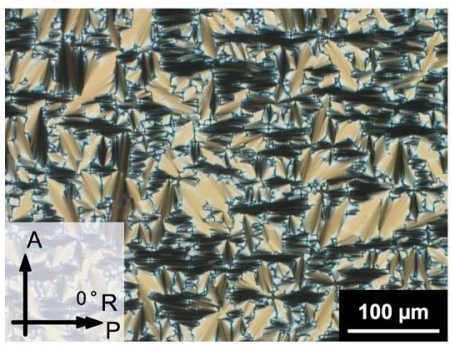

(b)

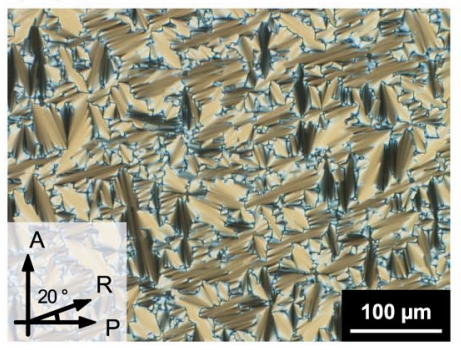

(c)

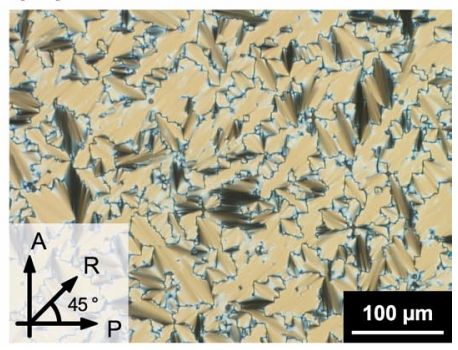

Figure S5. Polarizing optical microscopic images of the aligned complexes of 1 containing 0.05 mol of lithium triflate per ethylene oxide unit on polyimide 4. The rubbing direction is at the angle of (a) $0^{\circ}$ (b) $20^{\circ}$ (c) $45^{\circ}$ to polarizer or analyzer under crossed Nicols condition. Directions of A: analyzer; P: polarizer; R: rubbing.

\section{Preparation of Line-Shaped ITO Substrates and LC Cells for Ionic Conductivity.}

Patterned ITO-coated glass substrates were prepared by following procedure. Photoresist was spincoated onto an ITO substrate and exposed to Hg lamp through photomask and developed with a 2.38 wt $\%$ aqueous tetramethylammonium hydroxide solution. Etching of ITO was performed with a $5 \mathrm{wt} \%$ aqueous solution of sulfuric acid and nitric acid (1/1) to pattern the ITO. Photoresist was stripped using $\mathrm{N}$-methyl-2-pyrrolidone leaving the desired pattern. Patterned substrates were washed in 2-propanol, acetone, aqueous detergent solution, and deionized water by ultrasonic cleaning.

The LC cells were assembled as shown in Figure 5a. The ionic conductivities were obtained across the LC film between alignment layers. Polyimides were coated and rubbed on line-shaped ITO substrates. Two lines of ITO cross perpendicularly. An alternating electric field is applied in the square area crossing the two ITO lines.

\section{References and Notes}

(1) (a) Lee, H. J.; Won, J.; Park, H. C.; Lee, H.; Kang, Y. S. J. Membr. Sci. 2000, 178, 35-41. (b) Tsujita, Y.; Tanaka, H.; Yoshimizu, H.; Kinoshita, T.; Abe, T.; Kohtoh, N. J. Appl. Polym. Sci. 1994, 54, 1297-1304. (c) Saeed, M. B.; Zhan, M.-S. Eur. Polym. J. 2006, 42, 1844-1854. 